\title{
STEADY FLOW OF NON-NEWTONIAN FLUIDS THROUGH TUBES*
}

\author{
BY \\ A. E. GREEN AND R. S. RIVLIN \\ Brown University
}

1. Introduction. The stress-rate of deformation relation for an isotropic fluid, in which the stress at any point is assumed to depend only on the velocity gradients at that point, has been considered by Reiner [1] ${ }^{* *}$ and by Rivlin [2]. If the fluid is incompressible and the stress components are expressible as polynomials in the velocity gradients only, then the stress tensor can be expressed, using Cartesian tensors, in the form

$$
t_{i j}=\theta d_{i j}+\Psi d_{i k} d_{k i}+p \delta_{i j}
$$

where $d_{i j}$ is the rate of deformation tensor defined in terms of the velocity vector $v_{i}$ by

$$
d_{i j}=\frac{1}{2}\left(v_{i, i}+v_{i, i}\right), \quad d_{i i}=0 .
$$

$p$ is an arbitrary hydrostatic pressure and a comma denotes differentiation with respect to one of the coordinates $x_{i}$. Also, $\theta$ and $\Psi$ are polynomials in the invariants $I I$ and $I I I$ defined by

$$
I I=\frac{1}{2} d_{i j} d_{i i}, \quad I I I=\frac{1}{3} d_{i i} d_{i k} d_{k i} .
$$

Rivlin [3] has discussed the steady rectilinear flow of a fluid, for which the stress is given by (1.1), through a uniform tube of circular cross-section and has shown that such a rectilinear flow can be maintained without the application of body forces, without making further assumptions regarding the form of the dependence of $\theta$ and $\Psi$ on $I I$ and

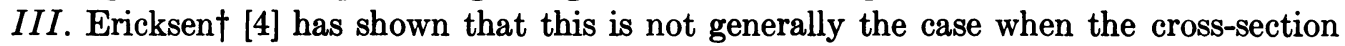
of the tube is non-circular and that, in such cases, the maintenance of steady rectilinear flow through the tube will require that, in addition to a uniform "pressure gradient" along the tube, an appropriate distribution of body forces be applied to the fluid. An exception occurs when $\Psi=k \theta$, where $k$ is a constant. In this case steady rectilinear flow of the fluid through the tube can be maintained by a uniform "pressure gradient" alone.

In the present paper, a suitable distribution of body forces, for the maintenance of steady rectilinear flow through a non-circular tube, is calculated. This distribution has non-zero components only in the planes normal to the direction of flow and these components are independent of position along the tube. This result suggests that if a uniform

*Received November 16, 1955. The results presented in this paper were obtained in the course of research sponsored by the Office of Ordnance Research, Department of the Army, under Contract No. DA-19-020-3487.

**Numbers in square brackets refer to the Bibliography at the end of the paper.

†We are grateful to Dr. J. L. Ericksen for discussing this result with us and for allowing us to see the typescript of his paper prior to publication. 
"pressure gradient" is applied to the fluid, and no body forces are applied, a steady flow will be produced which consists of a rectilinear flow along the tube on which is superposed some flow distribution in planes perpendicular to the length of the tube, independent of position along the tube. This flow distribution is calculated for a tube having an elliptical cross-section, for a fluid whose stress-rate of deformation relation has the form (1.1) with $\theta$ constant and $\Psi=\alpha(c+I I)$, where $c$ is a constant and where $\alpha$ is a sufficiently small constant so that the governing equations determining the flow can be linearized with respect to $\alpha$. This fluid is a nearly Newtonian fluid which departs from the ideal Newtonian law (obtained by taking $\alpha=0$ ) in a particular way. The choice of this particular form of departure is governed by considerations of mathematical tractability. It is found that for such a fluid, a uniform pressure gradient will produce in the elliptical tube a rectilinear flow on which is superposed four similar vortex-like flows in planes normal to the length of the tube-one in each quadrant of the elliptical cross-section. These have the same signs in diagonally opposite quadrants and opposite signs in adjacent quadrants. Although the quantitative results have been obtained for a particular type of non-Newtonian fluid, we may expect them to be qualitatively valid much more generally.

2. Rectilinear flow. Assuming zero body forces Ericksen [4] has examined steady flow for which the velocity components are

$$
v_{1}=v_{2}=0, \quad v_{3}=2 f(x, y),
$$

in a fluid for which the stress-rate of deformation relation is given by (1.1), where coordinates $x_{i}$ are replaced by $x, y, z$. Here we reconsider flow given by (2.1) but retain, at present, body forces. From (1.1) and (2.1) we have

$$
\begin{gathered}
d_{i j}=\left\|\begin{array}{ccc}
0, & 0, & f_{x} \\
0, & 0, & f_{y} \\
f_{x}, & f_{y}, & 0
\end{array}\right\|, \\
d_{i r} d_{r i}=\left\|\begin{array}{ccc}
f_{x}^{2}, & f_{x} f_{y}, & 0 \\
f_{x} f_{y}, & f_{y}^{2}, & 0 \\
0, & 0, & f_{x}^{2}+f_{y}^{2}
\end{array}\right\|,
\end{gathered}
$$

where suffixes denote partial differentiation. Also, from (1.1), (1.3), (2.2), (2.3) it follows that

$$
\begin{aligned}
& I I=f_{x}^{2}+f_{y}^{2}, \quad I I I=0, \\
& t_{11}=\Psi f_{x}^{2}+p, \quad t_{12}=\Psi f_{x} f_{y}, \quad t_{22}=\Psi f_{y}^{2}+p, \\
& t_{13}=\theta f_{x}, \quad t_{23}=\theta f_{y}, \quad t_{33}=\Psi\left(f_{x}^{2}+f_{y}^{2}\right)+p .
\end{aligned}
$$

If we denote body force components by $(X, Y, Z)$ and the (constant) density by $\rho$, the usual equations of motion reduce to 


$$
\begin{gathered}
\rho X+\frac{\partial p}{\partial x}+\frac{\partial}{\partial x}\left(\Psi f_{x}^{2}\right)+\frac{\partial}{\partial y}\left(\Psi f_{x} f_{y}\right)=0 \\
\rho Y+\frac{\partial p}{\partial y}+\frac{\partial}{\partial x}\left(\Psi f_{x} f_{y}\right)+\frac{\partial}{\partial y}\left(\Psi f_{y}^{2}\right)=0 \\
\rho Z+\frac{\partial p}{\partial z}+\frac{\partial}{\partial x}\left(\theta f_{x}\right)+\frac{\partial}{\partial y}\left(\theta f_{y}\right)=0
\end{gathered}
$$

if we use (2.5) and observe that inertia terms for the flow (2.1) vanish.

We now seek for a solution in which $Z$ is zero and $X, Y$ depend only on $x$ and $y$. It follows from the third of equations (2.6) that

$$
p=p_{0}(x, y)+\lambda Z
$$

where $\lambda$ is a constant such that

$$
\frac{\partial}{\partial x}\left(\theta f_{x}\right)+\frac{\partial}{\partial y}\left(\theta f_{y}\right)+\lambda=0 .
$$

The function $\Psi$ depends only on the invariant $I I$ which is given by (2.4). Hence, writing

$$
q=p_{0}+\frac{1}{2} \int \Psi d I I
$$

we have

$$
\begin{aligned}
& \frac{\partial q}{\partial x}=\frac{\partial p_{0}}{\partial x}+\Psi\left(f_{x} f_{x x}+f_{y} f_{x y}\right), \\
& \frac{\partial q}{\partial y}=\frac{\partial p_{0}}{\partial y}+\Psi\left(f_{x} f_{x y}+f_{y} f_{y y}\right) .
\end{aligned}
$$

It then follows that the first two of Eqs. (2.6) may be rewritten in the form

$$
\begin{aligned}
& \rho X+\frac{\partial q}{\partial x}+\left[\frac{\partial}{\partial x}\left(\Psi f_{x}\right)+\frac{\partial}{\partial y}\left(\Psi f_{y}\right)\right] f_{x}=0 \\
& \rho Y+\frac{\partial q}{\partial y}+\left[\frac{\partial}{\partial x}\left(\Psi f_{x}\right)+\frac{\partial}{\partial y}\left(\Psi f_{y}\right)\right] f_{y}=0 .
\end{aligned}
$$

Ericksen [4] has shown that when $X$ and $Y$ are zero, Eqs. (2.10) imply that $q$ is a function of $f$ only, and, in general, they are incompatible with Eq. (2.8) for $f$. When $X, Y$ are non-zero a possible solution of Eqs. (2.10) is

$$
\begin{aligned}
& \rho X=-\left[\frac{\partial}{\partial x}\left(\Psi f_{x}\right)+\frac{\partial}{\partial y}\left(\Psi f_{y}\right)+\frac{d q}{d f}\right] f_{x}, \\
& \rho Y=-\left[\frac{\partial}{\partial x}\left(\Psi f_{x}\right)+\frac{\partial}{\partial y}\left(\Psi f_{y}\right)+\frac{d q}{d f}\right] f_{y},
\end{aligned}
$$

where $q$ is now a function of $f$ only. The flow given by (2.1), where $f$ satisfies the differential equation (2.8), together with a suitable boundary condition, can then be maintained 
provided we apply body forces whose components $(X, Y)$ along the $(x, y)$ axes respectively, are given by (2.11).

From (2.8) and (2.11) it is seen that the body forces vanish when

(a) $\Psi=k \theta, q=k \lambda f$, where $k$ is a constant, or

(b) $f$ is a function of a linear combination of $x$ and $y$, or

(c) $f$ is a function of $\left(x^{2}+y^{2}\right)^{\frac{1}{2}}$,

in agreement with Ericksen [4].

If the boundary of the cross-section of the tube is symmetrical about both the $x$ and $y$-axes, we see that equation (2.8) for $f$, together with the condition that $f=0$ on the boundary, implies that $f$ is an even function of $x$ and an even function of $y$ ( $\theta$ depends only on $f_{x}^{2}+f_{y}^{2}$ ). Since $d q / d f$ in (2.11) is arbitrary we see that, in general, the flow may be maintained by a body force $X$ along the $x$-axis which is an odd function of $x$ and an even function of $y$; and by a body force $Y$ which is an even function of $x$ and an odd function of $y$. This suggests that rectilinear flow down such a tube may be possible without body forces, if in addition there is a flow in a perpendicular direction in each quadrant of the $x y$ plane, the flow in any one quadrant being the mirror image of that in the immediately adjacent quadrants. In Sec. 5 this is confirmed for a special type of fluid flowing in a tube of elliptical cross-section.

Suppose now that the fluid is such that the restriction (a) is satisfied approximately. We put

$$
\Psi=k \theta+\alpha \Psi^{\prime}, \quad q=k \lambda f,
$$

and Eqs. (2.11) and (2.8) then yield

$$
\begin{aligned}
& \rho X=-\alpha\left\{\frac{\partial}{\partial x}\left(\Psi^{\prime} f_{x}\right)+\frac{\partial}{\partial y}\left(\Psi^{\prime} f_{y}\right)\right\} f_{x}, \\
& \rho Y=-\alpha\left\{\frac{\partial}{\partial x}\left(\Psi^{\prime} f_{x}\right)+\frac{\partial}{\partial y}\left(\Psi^{\prime} f_{y}\right)\right\} f_{y},
\end{aligned}
$$

where $\Psi^{\prime}$ is a function of the invariant $I I$, and $\alpha$ is a sufficiently small parameter. The value of $q$ can be chosen arbitrarily and we choose to take the particular value in (2.12). In general, therefore, body forces $(X, Y)$ of order $\alpha$ are required to maintain the flow (2.1). For example, if the fluid is nearly Newtonian in such a way that

$$
\theta=2 \mu
$$

then $Y, Y$ are $0(\alpha)$.

In the next section we examine the modifications which are required in order to obtain steady flow in pipes, when body forces are zero, for a general fluid for which Eq. (1.1) holds.

3. General steady flow in tubes. We seek a solution of the fundamental equations, with zero body forces, in which all the velocity components are independent of time $t$ and the coordinate $z$. We therefore introduce into (2.1) non-zero values of $v_{1}, v_{2}$. In view of the incompressibility condition $d_{i i}=0$ we may write

$$
v_{1}=-\psi_{\nu}, \quad v_{2}=\psi_{x}, \quad v_{3}=2 f,
$$


$\psi$ and $f$ being functions of $x, y$ only. From (1.2) and (3.1) we have

$$
\begin{gathered}
d_{i i}=\left\|\begin{array}{crc}
-\psi_{x y}, & \frac{1}{2}\left(\psi_{x x}-\psi_{y y}\right), & f_{x} \\
\frac{1}{2}\left(\psi_{x x}-\psi_{y y}\right), & \psi_{x y}, & f_{y} \\
f_{x}, & f_{y}, & 0
\end{array}\right\| . \\
d_{1 m} d_{m 1}=\psi_{x y}^{2}+\frac{1}{4}\left(\psi_{x x}-\psi_{y y}\right)^{2}+f_{x}^{2}, \\
d_{1 m} d_{m 2}=f_{x} f_{y}, \\
d_{1 m} d_{m 3}=-f_{x} \psi_{x y}+\frac{1}{2} f_{\nu}\left(\psi_{x x}-\psi_{y \nu}\right) \\
d_{2 m} d_{m 2}=\psi_{x y}^{2}+\frac{1}{4}\left(\psi_{x x}-\psi_{y \nu}\right)^{2}+f_{y}^{2} \\
d_{2 m} d_{m 3}=f_{y} \psi_{x y}+\frac{1}{2} f_{x}\left(\psi_{x x}-\psi_{y y}\right) \\
d_{3 m} d_{m 3}=f_{x}^{2}+f_{y}^{2},
\end{gathered}
$$

and the strain invariants (1.3) are then

$$
\begin{gathered}
I I=f_{x}^{2}+f_{y}^{2}+\psi_{x y}^{2}+\frac{1}{4}\left(\psi_{x x}-\psi_{y y}\right)^{2}, \\
I I I=\left(f_{y}^{2}-f_{x}^{2}\right) \psi_{x y}+f_{x} f_{y}\left(\psi_{x x}-\psi_{y y}\right) .
\end{gathered}
$$

From (1.1), (3.2) and (3.3) we see that the components of stress are

$$
\begin{aligned}
& t_{11}=-\Theta \psi_{x y}+\Psi\left\{\psi_{x y}^{2}+\frac{1}{4}\left(\psi_{x x}-\psi_{y y}\right)^{2}+f_{x}^{2}\right\}+p, \\
& t_{12}=\frac{1}{2} \Theta\left(\psi_{x x}-\psi_{y y}\right)+\Psi f_{x} f_{y}, \\
& t_{22}=\theta \psi_{x y}+\Psi\left\{\psi_{x y}^{2}+\frac{1}{4}\left(\psi_{x x}-\psi_{y y}\right)^{2}+f_{y}^{2}\right\}+p, \\
& t_{13}=\theta f_{x}+\Psi\left\{-f_{x} \psi_{x y}+\frac{1}{2} f_{y}\left(\psi_{x x}-\psi_{y y}\right)\right\}, \\
& t_{23}=\theta f_{y}+\Psi\left\{f_{y} \psi_{x y}+\frac{1}{2} f_{x}\left(\psi_{x x}-\psi_{y y}\right)\right\}, \\
& t_{33}=\Psi\left(f_{x}^{2}+f_{y}^{2}\right)+p .
\end{aligned}
$$

Using (3.1) and (3.5) we may now write down the equations of motion. A more convenient form of these equations may, however, be obtained by introducing the complex variables $\zeta, \zeta^{*}$ where

$$
\zeta=x+i y, \quad \zeta^{*}=x-i y .
$$

Then, observing that

$$
\frac{\partial}{\partial x}=\frac{\partial}{\partial \zeta}+\frac{\partial}{\partial \zeta^{*}}, \quad \frac{\partial}{\partial y}=i\left(\frac{\partial}{\partial \zeta}-\frac{\partial}{\partial \zeta^{*}}\right),
$$


we find, from (3.5), that

$$
\begin{aligned}
& t_{11}+t_{22}=2 p+4 \Psi\left(f_{\zeta} f_{\zeta^{*}}+2 \psi_{\zeta \zeta} \psi_{\zeta^{*} \zeta^{*}}\right), \\
& t_{11}-t_{22}+2 i t_{12}=4 i \Theta \psi_{\zeta^{*} \zeta^{*}}+4 \Psi f_{\zeta^{*}}^{2}, \\
& t_{13}+i t_{23}=2 \Theta f_{\zeta^{*}}+4 i \Psi f_{\zeta} \psi_{\zeta^{*} \zeta^{*}}, \\
& t_{33}=p+4 \Psi f_{\zeta} f_{\zeta^{*}} .
\end{aligned}
$$

Also, using (3.1) and (3.6), the equations of motion become

$$
\begin{gathered}
\frac{\partial}{\partial \zeta^{*}}\left(t_{11}+t_{22}\right)+\frac{\partial}{\partial \zeta}\left(t_{11}-t_{22}+2 i t_{12}\right)=4 \rho\left(\psi_{\zeta} \psi_{\zeta * \zeta *}-\psi_{\zeta *} \psi_{\zeta \zeta^{*}}\right), \\
\frac{\partial t_{33}}{\partial z}+\frac{\partial}{\partial \zeta}\left(t_{13}+i t_{23}\right)+\frac{\partial}{\partial \zeta^{*}}\left(t_{13}-i t_{23}\right)=4 i \rho\left(f_{\zeta} \psi_{\zeta *}-f_{\zeta *} \psi_{\zeta}\right),
\end{gathered}
$$

so that with (3.8) we then have

$$
\begin{aligned}
& \frac{\partial p}{\partial \zeta^{*}}+2 \frac{\partial}{\partial \zeta^{*}}\left\{\Psi\left(f_{\zeta} f_{\zeta^{*}}+2 \psi_{\zeta \zeta} \psi_{\zeta^{*} \zeta^{*}}\right)\right\} \\
&+2 \frac{\partial}{\partial \zeta}\left\{i \theta \psi_{\zeta^{*} \zeta^{*}}+\Psi f_{\zeta^{*}}^{2}\right\}=2 \rho\left(\psi_{\zeta} \psi_{\zeta^{*} \zeta^{*}}-\psi_{\zeta *} \psi_{\zeta \zeta^{*}}\right) \\
& \frac{\partial p}{\partial z}+2 \frac{\partial}{\partial \zeta}\left\{\theta f_{\zeta^{*}}+2 i \Psi f_{\zeta} \psi_{\zeta^{*} \zeta^{*}}\right\} \\
&+2 \frac{\partial}{\partial \zeta^{*}}\left\{\theta f_{\zeta}-2 i \Psi f_{\zeta^{*}} \psi_{\zeta \zeta}\right\}=4 i \rho\left(f_{\zeta} \psi_{\zeta^{*}}-f_{\zeta^{*}} \psi_{\zeta}\right)
\end{aligned}
$$

From these equations we see that

$$
p=\lambda z+p_{0}\left(\zeta, \zeta^{*}\right)
$$

where $\lambda$ is a constant and $p_{0}$ is a function of $\zeta, \zeta^{*}$. Hence

$$
\begin{gathered}
\lambda+2 \frac{\partial}{\partial \zeta}\left\{\theta f_{\zeta^{*}}+2 i \Psi f_{\zeta} \psi_{\zeta^{*} \zeta^{*}}\right\}+2 \frac{\partial}{\partial \zeta^{*}}\left\{\theta f_{\zeta}-2 i \Psi f_{\zeta^{*}} \psi_{\zeta \zeta}\right\} \\
\frac{\partial p_{0}}{\partial \zeta^{*}}+2 \frac{\partial}{\partial \zeta^{*}}\left\{\Psi\left(f_{\zeta} f_{\zeta^{*}}+2 \psi_{\zeta \zeta} \psi_{\zeta^{*} \zeta^{*}}\right)\right\} \\
+2 \frac{\partial}{\partial \zeta}\left\{i \theta \psi_{\zeta^{*} \zeta^{*}}+\Psi f_{\zeta^{*}}-f_{\zeta^{*}}\right\}=2 \rho\left(\psi_{\zeta}\right) \\
\left.+\psi_{\zeta^{*} \zeta^{*}}-\psi_{\zeta^{*}} \psi_{\zeta \zeta^{*}}\right) .
\end{gathered}
$$

We also note that the invariants in (3.4) may now be written in the form

$$
\begin{gathered}
I I=4\left(f_{\zeta} f_{\zeta^{*}}+\psi_{\zeta \zeta} \psi_{\zeta^{*} \zeta^{*}}\right), \\
I I I=4 i\left(f_{\zeta^{*}}^{2} \psi_{\zeta^{*} \zeta^{*}}-f_{\zeta^{*}}^{2} \psi_{\zeta \zeta}\right) .
\end{gathered}
$$

Equations (3.13) and (3.14) are three equations for the three unknown functions $p_{0}, f, \psi$. In addition, if we impose the condition that the velocity is zero on some closed 
curve $G\left(\zeta, \zeta^{*}\right)=0$ in the $(x, y)$ plane then

$$
f=0, \quad \psi_{\zeta}=0 \quad\left[G\left(\zeta, \zeta^{*}\right)=0\right] .
$$

In the next section we consider the solution of Eqs. (3.13) and (3.14), subject to boundary conditions (3.16), for a fluid for which

$$
\Psi=k \theta+\alpha \Psi^{\prime} .
$$

This fluid was discussed in Sec. 2 where it was seen that, when $\alpha=0$, a solution of the problem was possible with $\psi=0$.

4. Solution by successive approximations. We suppose that $\Psi$ is given by (3.17). We seek a solution of Eqs. (3.13) and (3.14) in power series in $\alpha$, based on the solution $\psi=0$ when $\alpha=0$, assuming that $\alpha$ is a parameter which is sufficiently small, but we only carry out detailed calculations for terms of $0(\alpha)$. Thus, we assume that

$$
\psi=\alpha \varphi+\cdots, \quad f=h+\alpha g+\cdots,
$$

so that, from (3.15)

$$
\begin{aligned}
I I & =I I_{0}+\alpha I I_{1}+\cdots, \\
I I I & =\alpha I I I_{1}+\cdots,
\end{aligned}
$$

where

$$
\begin{gathered}
I I_{0}=4 h_{\zeta} h_{\zeta^{*}}, \quad I I_{1}=4\left(h_{5} g_{\zeta^{*}}+h_{\zeta^{*}} g_{\zeta}\right), \\
I I I_{1}=4 i\left(h_{\zeta}^{2} \varphi_{\zeta^{*} \zeta^{*}}-h_{\zeta^{*}}^{2} \varphi_{\zeta \zeta}\right) .
\end{gathered}
$$

Assuming that $\theta$ and $\Psi^{\prime}$ are analytic functions of $\alpha$, we then have

$$
\begin{aligned}
\Theta(I I, I I I) & =\Theta_{0}+\alpha \theta_{1}+\cdots, \\
\Psi^{\prime}(I I, I I I) & =\Psi_{0}^{\prime}+\cdots,
\end{aligned}
$$

where

$$
\begin{aligned}
& \Theta_{0}=\Theta\left(I I_{0}, 0\right), \quad \Psi_{0}^{\prime}=\Psi^{\prime}\left(I I_{0}, 0\right), \\
& \Theta_{1}=I I_{1} \frac{\partial \Theta\left(I I_{0}, 0\right)}{\partial I I}+I I I_{1} \frac{\partial \theta\left(I I_{0}, 0\right)}{\partial I I I} .
\end{aligned}
$$

Hence, from (3.17) and (4.4),

$$
\Psi=k \Theta_{0}+\alpha\left(k \Theta_{1}+\Psi_{0}^{\prime}\right)+\cdots .
$$

We now substitute (4.1), (4.4) and (4.6) into Eqs. (3.13) and (3.14), and equate coefficients of powers of $\alpha$. It follows that the constant $\lambda$ has the form

$$
\lambda=\lambda_{0}+\alpha \lambda_{1}+\cdots,
$$

where $\lambda_{0}, \lambda_{1}, \cdots$ are constants. Also, if we recall (2.9) and result (a) in Sec. 2, we see that the pressure may be written

$$
p_{0}=-\frac{k}{2} \int \Theta_{0} d I I_{0}+k \lambda_{0} h+\alpha p_{1}+\cdots,
$$


where $p_{1}$ is a function of $\zeta, \zeta^{*}$ only. In addition, using (4.7) and (4.8), we see that the terms independent of $\alpha$ in (3.13) yield the equation

$$
2\left\{\frac{\partial}{\partial \zeta}\left(\theta_{0} h_{\zeta^{*}}\right)+\frac{\partial}{\partial \zeta^{*}}\left(\theta_{0} h_{\zeta}\right)\right\}+\lambda_{0}=0,
$$

whilst the terms independent of $\alpha$ in (3.14) now cancel. If we consider terms in $\alpha$ in (3.13) and (3.14) we obtain

$$
\begin{aligned}
\lambda_{1} & +2 \frac{\partial}{\partial \zeta}\left\{\theta_{0} g_{\zeta^{*}}+\theta_{1} h_{\zeta^{*}}+2 i k \theta_{0} h_{\zeta \varphi_{\zeta *}}\right\} \\
& +2 \frac{\partial}{\partial \zeta^{*}}\left\{\theta_{0} g_{\zeta}+\theta_{1} h_{\zeta}-2 i k \theta_{0} h_{\zeta * \varphi_{\zeta \zeta}}\right\}=4 i \rho\left(h_{\zeta} \varphi_{\zeta^{*}}-h_{\zeta^{*} \varphi_{\zeta}}\right) \\
\frac{\partial p_{1}}{\partial \zeta^{*}} & +2 \frac{\partial}{\partial \zeta^{*}}\left\{k \theta_{0}\left(h_{\zeta} g_{\zeta^{*}}+h_{\zeta *} g_{\zeta}\right)+\left(k \theta_{1}+\Psi_{0}^{\prime}\right) h_{\zeta} h_{\zeta^{*}}\right\} \\
& +2 \frac{\partial}{\partial \zeta}\left\{i \theta_{0} \varphi_{\zeta^{*} \zeta^{*}}+2 k \theta_{0} h_{\zeta^{*}} g_{\zeta^{*}}+\left(k \theta_{1}+\Psi_{0}^{\prime}\right) h_{\zeta^{*}}^{2}\right\}=0
\end{aligned}
$$

We continue discussion of these equations for the special fluid which is defined by

$$
\theta=2 \mu, \quad \Psi^{\prime}=c+I I, \quad k=0,
$$

where $\mu$ and $c$ constants, so that

$$
\theta_{0}=2 \mu, \quad \Psi_{0}^{\prime}=c+I I_{0}, \quad k=0, \quad \theta_{1}=0 .
$$

Equations (4.9), (4.11) and (4.10) respectively then reduce to

$$
\begin{gathered}
8 \mu h_{\zeta \zeta^{*}}+\lambda_{0}=0 \\
\frac{\partial p_{1}}{\partial \zeta^{*}}+4 i \mu \varphi_{\zeta \zeta^{*}}+8 \frac{\partial}{\partial \zeta^{*}}\left(h_{\zeta}^{2} h_{\zeta^{*}}^{2}\right)+8 \frac{\partial}{\partial \zeta}\left(h_{\zeta} h_{\zeta^{*}}^{3}\right)+2 c\left\{\frac{\partial}{\partial \zeta^{*}}\left(h_{\zeta} h_{\zeta^{*}}\right)+\frac{\partial}{\partial \zeta}\left(h_{\zeta^{*}}^{2}\right)\right\}=0 \\
8 \mu g_{\zeta \zeta^{*}}+\lambda_{1}=4 i \rho\left(h_{\zeta \varphi_{\zeta *}}-h_{\zeta^{*}} \varphi_{\zeta}\right) .
\end{gathered}
$$

If we differentiate Eq. (4.15) with respect to $\zeta$, subtract the result from its complex conjugate, and use (4.14), we obtain the following equation for $\varphi$ :

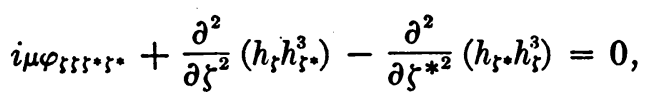

or

$$
\begin{aligned}
i \mu \varphi_{\zeta \zeta \zeta^{*}} & +6 h_{\zeta \zeta^{*}}\left(h_{\zeta *}^{2} h_{\zeta \zeta}-h_{\zeta}^{2} h_{\zeta * \zeta *}\right) \\
& +h_{\zeta *}^{3} h_{\zeta \zeta \zeta}-h_{\zeta \zeta^{3} h^{*} \zeta^{*}} \\
& +3 h_{\zeta} h_{\zeta *}\left(h_{\zeta *} h_{\zeta \zeta \zeta^{*}}-h_{\zeta} h_{\zeta \zeta^{*}}\right)=0 .
\end{aligned}
$$

5. Flow through elliptical pipe. In this section we consider the solution of Eqs. (4.14), (4.16) and (4.17) for the special case of flow through a pipe whose cross-section is the ellipse

$$
\frac{x^{2}}{a^{2}}+\frac{y^{2}}{b^{2}}=1
$$


In complex coordinates $\zeta, \zeta^{*}$ this equation may be re-written in the form

$$
\left(a^{2}+b^{2}\right) \zeta \zeta^{*}-\frac{1}{2}\left(a^{2}-b^{2}\right)\left(\zeta^{2}+\zeta^{* 2}\right)-2 a^{2} b^{2}=0 .
$$

At this boundary we assume that the velocity of the fluid is zero so that

$$
h=0, \quad \varphi_{\zeta}=0, \quad g=0,
$$

when $\zeta, \zeta^{*}$ are related by Eq. (5.2).

Since $h$ vanishes on the boundary (5.2) we try a solution of (4.14) of the form

$$
h=A\left\{\left(a^{2}+b^{2}\right) \zeta \zeta^{*}-\frac{1}{2}\left(a^{2}-b^{2}\right)\left(\zeta^{2}+\zeta^{*^{2}}\right)-2 a^{2} b^{2}\right\} .
$$

This satisfies Eq. (4.14) if

$$
8 \mu\left(a^{2}+b^{2}\right) A=-\lambda_{0} .
$$

If we substitute $h$ from (5.4) in the right hand side of (4.17) we have

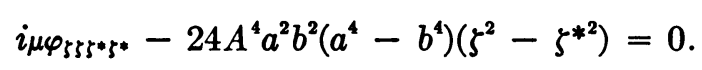

We require a solution of (5.6) such that $\varphi_{\zeta}$ (and therefore $\varphi_{\zeta^{*}}$ ) vanishes on the boundary (5.2). We therefore assume that

$$
i_{\mu \varphi}=B\left\{\left(a^{2}+b^{2}\right) \zeta \zeta^{*}-\frac{1}{2}\left(a^{2}-b^{2}\right)\left(\zeta^{2}+\zeta^{*^{2}}\right)-2 a^{2} b^{2}\right\}^{2}\left(\zeta^{2}-\zeta^{*^{2}}\right) .
$$

This satisfies the boundary conditions and will also satisfy the differential equation (5.6) if

$$
B\left\{4\left(a^{2}+b^{2}\right)^{2}+\left(a^{2}-b^{2}\right)^{2}\right\}-4 A^{4} a^{2} b^{2}\left(a^{4}-b^{4}\right)=0 .
$$

Using (5.8), and expressing $\varphi$ in terms of the coordinates $x, y$, we have

$$
\varphi=\frac{64 A^{4} a^{2} b^{2}\left(a^{4}-b^{4}\right)\left(b^{2} x^{2}+a^{2} y^{2}-a^{2} b^{2}\right)^{2} x y}{\mu\left\{4\left(a^{2}+b^{2}\right)^{2}+\left(a^{2}-b^{2}\right)^{2}\right\}} .
$$

This represents a flow in the $x, y$ plane whose streamlines are the curves $\varphi=$ constant. The flow is illustrated in Fig. 1.

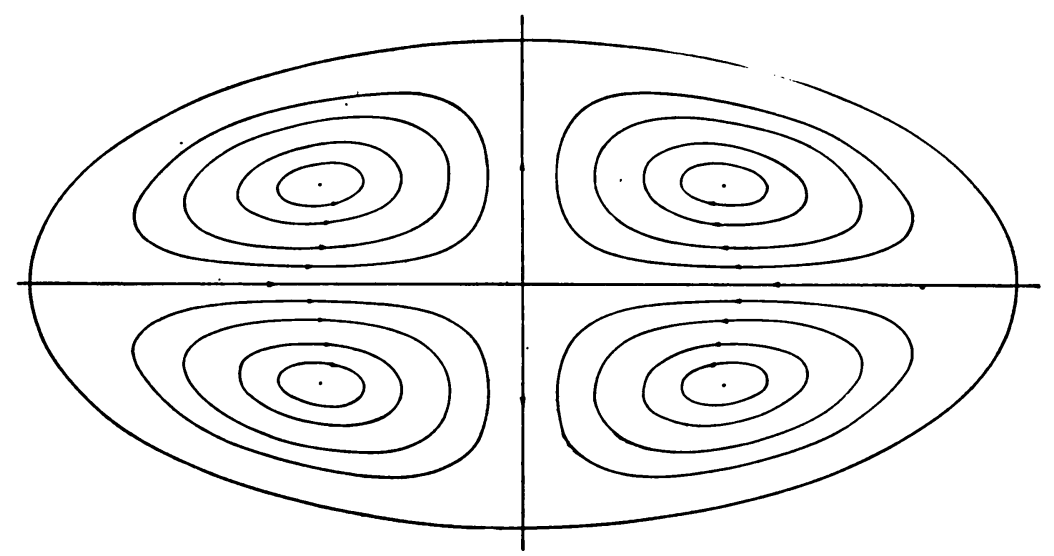

Fia. 1. Secondary flow in a tube with elliptical cross-section $(\alpha>0)$. 
To complete the solution of the problem as far as terms of order $\alpha$ are concerned we must now solve Eq. (4.16). In this equation we may put $\lambda_{1}=0$ since this only contributes terms of the same type as those in the original flow down the tube. The remaining terms, which arise solely from the inertia of the material, are found from (4.16), where $h, \varphi$ are given by (5.4) and (5.7) respectively. Thus

$$
\begin{aligned}
8 \mu g_{\zeta \zeta^{*}}=\frac{8 \rho A B}{\mu}\left\{\left(a^{2}+b^{2}\right) \zeta \zeta^{*}\right. & -\frac{1}{2}\left(a^{2}-b^{2}\right)\left(\zeta^{2}+\zeta^{*^{2}}\right) \\
& \left.-2 a^{2} b^{2}\right\}^{2}\left\{2\left(a^{2}-b^{2}\right) \zeta \zeta^{*}\right. \\
& \left.-\left(a^{2}+b^{2}\right)\left(\zeta^{2}+\zeta^{*^{2}}\right)\right\},
\end{aligned}
$$

together with the boundary condition

$$
g=0,
$$

on the boundary (5.2). The solution of this boundary value problem is straightforward, but we omit details since the resulting flow will only modify slightly the original flow down the tube.

\section{REFERENCES}

1. M. Reiner, Amer. J. Math 67, 350-362 (1945)

2. R. S. Rivlin, Proc. Roy. Soc. A193, 260-281 (1948)

3. R. S. Rivlin, Proc. Cambridge Phil. Soc. 45, 88-91 (1949)

4. J. L. Ericksen, Quart. Appl. Math., in the press 